\title{
Fetale Mortalität bei Einlingen ab Termin - eine Analyse bundesdeutscher Perinataldaten 2004-2013
}

\section{Foetal Mortality in Singleton Foetuses at and beyond Term - An Analysis of German Perinatal Data 2004-2013}

\author{
Autoren \\ C. Schwarz ${ }^{1}$, E. Weiss ${ }^{2}$, C. Loytved ${ }^{3}$, R. Schäfers ${ }^{4}$, T. König ${ }^{5}$, P. Heusser ${ }^{6}$, B. Berger $^{6}$ \\ Institute \\ Die Institutsangaben sind am Ende des Beitrags gelistet
}

\section{Schlüsselwörter \\ - fetale Mortalität \\ Geburtseinleitung \\ - Totgeburt}

Key words

foetal mortality

induction of labour

stillbirth eingereicht $\quad 02.12 .2014$ angenommen 14.01.2015 nach Überarbeitung

\section{Bibliografie}

DOI http://dx.doi.org/ 10.1055/s-0034-1398659

Online-Publikation: 13.3.2015

Z Geburtsh Neonatol 2015;

219: $81-86$

(c) Georg Thieme Verlag KG Stuttgart · New York ISSN 0948-2393

\section{Korrespondenzadresse}

\section{Christiane Schwarz}

Frauenklinik AG Hebammenwissenschaft

Medizinische Hochschule Hannover

Carl-Neuberg-Straße 1

30625 Hannover

Tel.: + 49/511/532 6971

Fax: $+49 / 511 / 53218521$

schwarz.christiane@mh-

hannover.de

\section{Zusammenfassung}

$\nabla$

Hintergrund und Fragestellung: Die fetale Mortalität steigt vermutlich nach Überschreiten des Geburtstermins an. Ob eine Geburt eingeleitet werden soll, oder ob es besser ist, beobachtend abzuwarten, sobald eine Schwangere den Geburtstermin erreicht hat, bleibt Gegenstand von fachlichen Diskussionen. Darüber hinaus gibt es unterschiedliche Ansätze zur Berechnung des Risikos für eine Totgeburt. Um eine angemessene Diskussion über die Risiken der Terminüberschreitung führen zu können, werden aktuelle relevante Daten benötigt.

Methode: In der vorliegenden Arbeit wird die fetale Mortalität von Einlingen ohne Fehlbildungen in Deutschland zwischen 2004 und 2013 während verschiedener Schwangerschaftswochen beschrieben. Die Risikoberechnung erfolgt nach der Berechnungsgrundlage von Totgeburten je 1000 Geburten der jeweiligen Schwangerschaftswoche (SSW) vs. der Berechnung nach dem „fetus-at-risk“ Modell, in dem Totgeburten je 1000 fortbestehende Schwangerschaften zu dieser SSW zugrunde gelegt werden. Der Gemeinsame Bundesausschuss genehmigte die Sonderauswertung. Ergebnisse: 5933117 Geburten erfüllten die Einschlusskriterien. Die fetale Mortalität je 1000 Geburten der SSW ist am niedrigsten zwischen $41+0$ und $41+6$ SSW $(0,7 / 1000)$. Danach steigt die Rate auf 2,3/1000 zwischen $42+0$ und $42+6$ SSW. Mit dem „fetus-at-risk“ Modell zeigt sich die niedrigste Mortalität zwischen $37+0$ und $39+6$ SSW bei etwa 0,2 Totgeburten je 1000 fortbestehender Schwangerschaften. Zwischen der $41+0$ und $41+6$ liegt die fetale Mortalität bei 0,6 , und in der folgenden Woche dann bei 2,3 je 1000 . Ab der $42+6$ SSW ergibt sich bei beiden Rechenarten eine Mortalitätsrate von 6,3/1 000. Schlussfolgerung: Die fetale Mortalität ist bis $41+6$ SSW insgesamt sehr niedrig. Eine Empfehlung zur routinemäßigen Einleitung vor diesem Zeitpunkt scheint nicht sinnvoll.

\section{Abstract}

$\nabla$

Background: Foetal mortality appears to increase in postmature pregnancies. There is debate on the optimal timing of induction of labour vs. watchful waiting. The literature on retrospective analyses of secondary data is inconclusive. Different approaches to calculate foetal risk exist. Recent and relevant data are needed in order to realise an appropriate discussion.

Material and Methods: Mortality in singleton foetuses in Germany, between 2004 and 2013 was analysed in relation to gestational age. Risk for foetal death is described comparing stillbirths per 1000 births at a particular gestational age (GA) vs. stillbirths per 1000 ongoing pregnancies ("fetus-at-risk" model). Access to German routine perinatal data was granted. We included all stillbirths in singleton foetuses with no malformations after $36+6$ weeks gestational age from 2004 until 2013.

Results: 5,933,117 births fulfilled our inclusion criteria. Foetal mortality per 1000 births during that week of pregnancy is lowest between $41+0$ and $41+6$ days of $(0.7 / 1000)$. Mortality then increases to $2.3 / 1000$ in $42+0$ to $42+6$ GA. With the "fetus-at-risk" model, mortality is low between $37+0$ and $39+6 \mathrm{GA}$, ranging from $0.2 / 1000$ ongoing pregnancies, increasing to $0.6 / 1000$ between $41+0$ and $41+6$, and 2.3/1000 in the following week. For pregnancies lasting longer than $42+6$ weeks, the stillbirth risk is identical at $6.3 / 1000$ with both calculation methods.

Conclusion: Fetal mortality is low until $41+6$ weeks of pregnancy. Interpretation of current data does not support a policy of routine IOL before this gestational age in singleton foetuses. 


\section{Einleitung und Fragestellung}

In den letzten Jahren wurde in Deutschland ebenso wie in anderen Ländern in Fachkreisen darüber debattiert, ob - und falls ja, wann - es sinnvoll sei, schwangeren Frauen nach Erreichen des Geburtstermins eine Geburtseinleitung zu empfehlen. Neben der Vermeidung einer fetalen Makrosomie mit möglicher Morbidität bei Mutter und/oder Kind ist ein wichtiges Anliegen einer derartig begründeten Geburtseinleitung, die perinatale Mortalität durch Vermeidung eines intrauterinen Fruchttods (IUFT) bei reifem Kind zu senken, da dieses Risiko nach retrospektiven Analysen ab 41+0 Schwangerschaftswochen (SSW) anzusteigen scheint [1]. Gülmezoglu et al. formulieren in der größten und aktuellsten Metaanalyse von publizierten Studien zu Perinataldaten eine number-needed-to-treat (NNT) von 410 Geburtseinleitungen ab $41+0$, die nötig sind, um einen perinatalen Todesfall zu verhindern [1]. Der Beweis für diese Annahme wurde bisher jedoch nicht erbracht. Welche ungeborenen Kinder tatsächlich von einer Geburtseinleitung profitieren könnten, bleibt unklar [2]. Ebenso gibt es unterschiedliche Erkenntnisse zur Frage, ob eine großzügige Indikation zur Einleitung die Kaiserschnittrate, die Häufigkeit der postpartalen Blutungen oder weitere unerwünschte Outcomes positiv beeinflussen [3-5]. Präferenzen von Schwangeren zur Geburtseinleitung wurden bisher nicht systematisch untersucht. Die adäquate Beratung von gesunden Schwangeren am Termin in Bezug auf die möglichen Vorgehensweisen (beobachtendes Abwarten oder Einleiten; falls ja, wann und wie) ist unter diesen Voraussetzungen kaum möglich. Schwangere Frauen möchten in Entscheidungsprozesse eingebunden werden, sie wünschen sich dafür Informationen über alle Optionen und deren Vor- und Nachteile in angemessener Form [6-9]. Auch die Interpretation der Daten, die den fachlichen Diskussionen zugrunde liegen, ist aus mehreren Gründen problematisch. Die geburtshilflichen, gesundheitspolitischen und sozialen Bedingungen aus den Datenerhebungszeiträumen der aktuellen Arbeiten sind nicht unbedingt auf die heutige Bundesrepublik übertragbar. Etliche Sekundärdaten, die den rezenten Arbeiten $[1,10]$ zugrunde liegen, sind nicht mehr aktuell; die Originalerhebungen für die jüngste Cochrane Metaanalyse [1] stammen aus den Jahren zwischen 1969 und 2005. Stock et al. [3] beziehen sich beim Vergleich von perinatalen Outcomes aus eingeleiteten und nicht eingeleiteten Geburten in Schottland (1981-2007) ebenfalls auf Daten, aus denen die Indikationen zur Einleitung für die Frauen, bei denen keine medizinischen Probleme dokumentiert sind, nicht mehr eindeutig nachvollziehbar sind. Darüber hinaus ist weder die genaue Bestimmung des voraussichtlichen Geburtstermins nachvollziehbar noch ist die jeweils zur Geburteinleitung angewendete klinische Vorgehensweise bei der Auswertung berücksichtigt [11]. Ein grundlegendes Problem liegt in der uneinheitlichen statistischen Berechnung fetaler Todesfallraten. Ohne eine Vereinheitlichung auf dieser Ebene kann die Frage zur Geburtseinleitung bei Terminüberschreitung nicht beantwortet werden. In vielen Publikationen, und dementsprechend in der Metaanalysen dieser Arbeiten, wird sowohl für die perinatale als auch für die fetale Mortalität die Inzidenz je 1000 (Lebend-)Geburten berechnet [1]. Hier gibt es allerdings die abweichende Empfehlung, wonach die fetale Mortalität nicht in Bezug auf 1000 Geburten, sondern auf 1000 noch bestehende Schwangerschaften zu Beginn des jeweils betrachteten Gestationszeitraums definiert wird [12-15]. Durch diese Berechnungsweise ergeben sich deutliche Abwei- chungen in den Ergebnissen und damit in der Interpretation und den daraus abgeleiteten Empfehlungen für die klinische Praxis. Doch selbst wenn dasselbe Berechnungsschema verwendet wird, deuten die Ergebnisse nicht in die gleiche Richtung. In ihrer vor Kurzem veröffentlichten Publikation stellen Weiss et al. anhand von aktuellen Daten aus Baden-Württemberg vor allem ab 41 + 0 SSW deutlich niedrigere Inzidenzen von fetalen Todesfällen im Vergleich zu den publizierten Daten aus anderen europäischen Ländern sowie den USA fest [15]. Die Autoren vermuten, dass sich diese Unterschiede möglicherweise dadurch erklären lassen, dass in Baden-Württemberg (ebenso wie in den anderen Bundesländern Deutschlands) mit der Überwachung der Feten mittels CTG bereits ab $40+0$ SSW in allen Fällen begonnen wird, während in den Vergleichsländern dieses fetale Monitoring erst ab $41+0$ SSW sporadisch und ab $42+0$ SSW routinemäßig durchgeführt wurde. Es bleiben also etliche offene Fragen angesichts dieser Publikationen und den daraus abgeleiteten Empfehlungen.

Die Fragestellung der vorliegenden Arbeit in Bezug auf die bundesdeutschen Perinataldaten 2004-2013 lautet vor diesem Hintergrund: Sind die bundesdeutschen Daten konsistent mit den baden-württembergischen von Weiss et al. 2014 publizierten Daten? Ist auch auf Bundesebene, und über einen längeren Zeitraum nachvollziehbar, dass die fetale Mortalität niedriger liegt als im internationalen Vergleich?

Eine interdisziplinäre Arbeitsgruppe der Universität Witten/ Herdecke führt aktuell im Rahmen der Erstellung einer Entscheidungshilfe „Geburtseinleitung bei Terminüberschreitung“ für schwangere Frauen dazu Recherchen durch. Die Arbeitsgruppe setzt sich dazu mit aktuellen perinatalen Daten auseinander. Die vorliegende Arbeit ist Teil des Projekts.

\section{Material und Methodik \\ $\nabla$}

Die perinatalen Daten der klinischen Geburtshilfe werden in Deutschland schon lange erhoben. Seit der gesetzlichen Festlegung der Qualitätssicherung in der Geburtshilfe im SGB V im Jahr 2001 wird die Steuerung und Publikation zentral über eine Bundesgeschäftsstelle vorgenommen (BQS-Bundesgeschäftsstelle Qualitätssicherung bis 2009, seitdem AQUA-Institut für angewandte Qualitätsförderung und Forschung im Gesundheitswesen $\mathrm{GmbH}$ ). Die Arbeitsgruppe beantragte unter der aufgeführten Fragestellung Daten aus der Qualitätsberichterstattung des Bundes beim AQUA-Institut. Einschlusskriterien waren alle Geburten von Einlingen ohne Fehlbildungen $>36+6$ Schwangerschaftswochen (SSW) in deutschen Kliniken von 2004-2013. Die Bewilligung der Sonderauswertung erfolgte durch den gemeinsamen Bundesausschuss. Die aggregierten Daten wurden vom AQUA Institut zur Verfügung gestellt. Eine Bereinigung der gelieferten Daten nach Plausibilität erfolgte durch das AQUA-Institut. Dafür wurden offensichtlich fehlerhaft dokumentierte Gestationsalter aus dem Datensatz entfernt. Als plausibel erachteten die Autorinnen und Autoren eine Schwangerschaftsdauer von 40+0 SSW mit Abweichungen von -119 Tagen (entspricht der vollendeten 23 . SSW) bis zu +28 Tagen (entspricht einer Terminüberschreitung von 4 Wochen). Zugrunde liegt die Annahme, dass unter $23+0$ SSW (-119 Tage) ein Geburtsgewicht von $>500 \mathrm{~g}$ unwahrscheinlich ist und somit eine solche frühe Totgeburt als Fehlgeburt dokumentiert wird. Ebenso kann angenommen werden, dass das Fortbestehen einer Schwangerschaft 
mit bekanntem Gestationsalter über 4 Wochen nach dem Stichtag 40+0 SSW äußerst unwahrscheinlich ist, und somit diese Fälle eher auf Dokumentationsfehlern beruhen.

Die Berechnung der fetalen Mortalität konnte auf der Grundlage der von Weiss et al. vorgeschlagenen statistischen Berechnung von Totgeburten je 1000 fortbestehenden Schwangerschaften mit freundlicher Unterstützung des Autors erfolgen, der seinen Berechnungsalgorithmus zur Verfügung stellte [15]. Gleichzeitig wurde zum Vergleich eine Berechnung der international und national verwendeten Bezugsgröße Totgeburten je 1000 Geburten in der jeweiligen Schwangerschaftswoche vorgenommen.

\section{Ergebnisse}

Die Rate an Geburtseinleitungen stieg von 2005 bis 2012 von $16,5 \%(n=109709)$ auf $21,9 \%(n=145265)$. Die Anzahl der in der Perinatalerhebung erfassten klinischen Geburten von Einlingen ohne Fehlbildungen mit einem Gestationsalter von mehr als $36+6$ SSW betrug nach Bereinigung von implausiblen Datensätzen im untersuchten Zeitraum von 2004-2013 genau 5933117 (zum Vergleich Baden-Württemberg 2004-2009: 472843). Die fetale Mortalität lag in den Jahren 2004-13 bei reifen Einlingen ohne Fehlbildungen bei 0,12\% (zum Vergleich Baden-Württemberg: 0,097\%). Wenn als Berechnungsschlüssel die statistische Bezugsgröße IUFT je 1000 Geburten zugrunde gelegt wird, sinkt das Risiko eines fetalen Todes rechnerisch zunächst mit wachsender Reife des Kindes und liegt in $41+0$ bis $41+6$ SSW am niedrigsten, um dann ab 41+6 SSW anzusteigen. Wird als statistische Bezugsgröße die Anzahl von Totgeburten je weiter fortbestehenden Schwangerschaften ausgewertet, zeigt sich im Zeitraum nach dem ET in der Woche $41+0$ bis $41+6$ SSW ebenfalls der niedrigste Wert und ab 41+6 SSW der Anstieg des Risikos. Allerdings bewirkt die Berechnungsart eine von 39 bis 37 SSW abfallende Rate von IUFT (vgl. Baden-Württemberg nach Weiss et al., $\odot$ Abb. 1).

Die Bundesdaten bestätigen die von Weiss et al. festgestellten Tendenzen [15]. Das gilt sowohl für die fetale Mortalität je 1000 Geburten der entsprechenden Schwangerschaftswoche als auch für die fetale Mortalitätsrate je 1000 fortbestehende Schwangerschaften ( $\bullet$ Abb. 2). Der Anstieg der fetalen Mortalität nach $41+6$ wird hier noch deutlicher als in Baden-Württemberg.

\section{Diskussion \\ $\nabla$}

Die Vor- und Nachteile von Geburtseinleitungen bei Schwangeren am und über dem errechneten Geburtstermin werden in der Literatur kontrovers diskutiert. Es besteht weitgehender Konsens darüber, dass die Rate an Totgeburten ab dem Termin ansteigt. Die logische Konsequenz scheint zu sein, die Schwangerschaft zu einem Zeitpunkt zu beenden, an dem eine Frühgeburt nicht mehr zu erwarten und das Risiko des intrauterinen Fruchttodes aufgrund von insuffizienter fetaler Versorgung bei überreifer

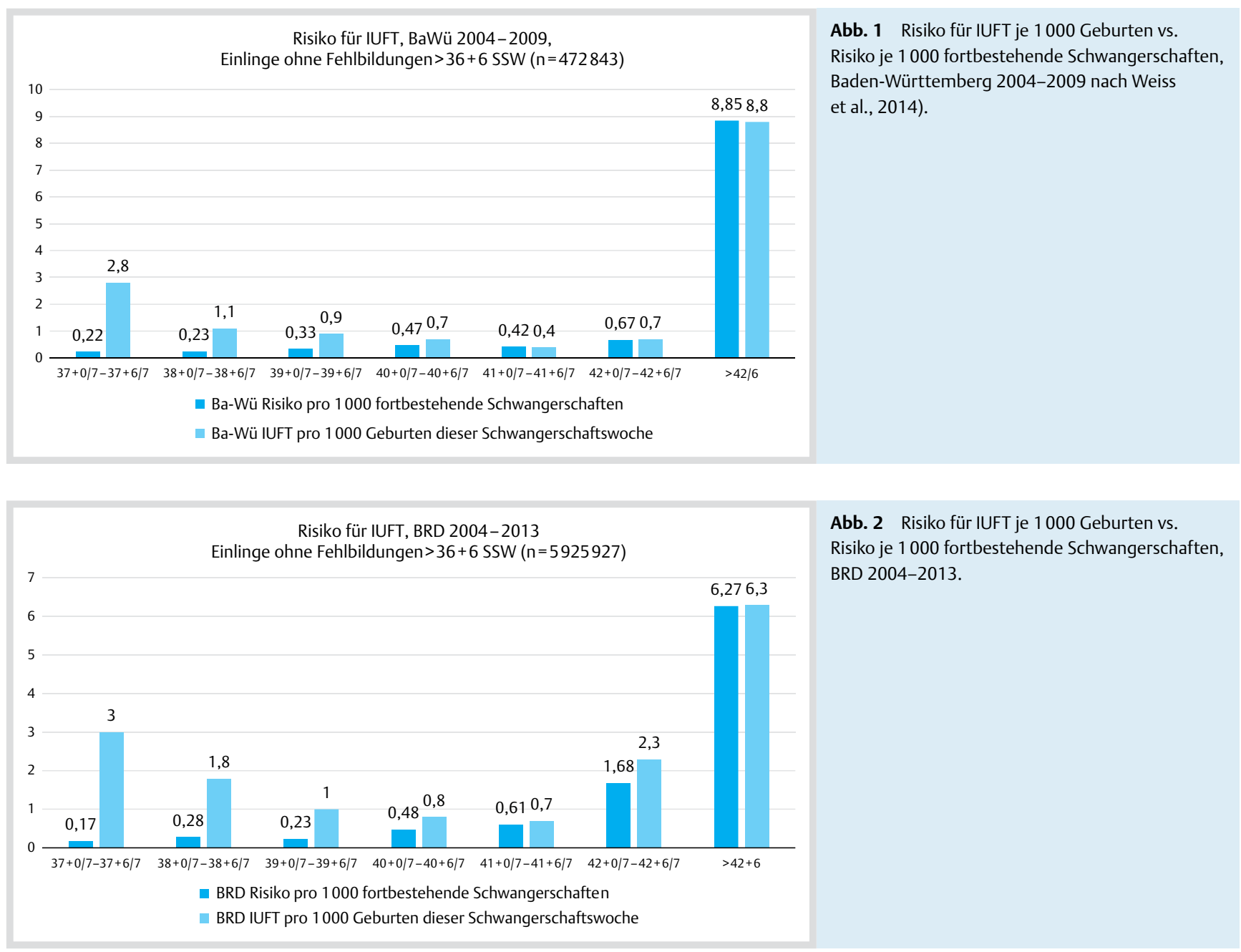


Plazenta noch moderat ist. Dieses Zeitfenster wird zwischen $41+0$ und 42 vollendeten Schwangerschaftswochen vermutet. Die Daten aus den berücksichtigten älteren Publikationen $[1,16,17]$ bestätigen diese Annahme. Es handelt sich allerdings dabei um Daten aus anderen Ländern, Gesundheitssystemen und anderen Kontexten medizinischer Versorgung sowie Lebensbedingungen. Die Anwendbarkeit und Übertragbarkeit der Schlussfolgerungen auf die heutige Bundesrepublik ist nicht selbstverständlich anzunehmen. Die Rate an IUFT liegt bei reifen Kindern bei 1,12 je 1000 Geburten ab 37+0 SSW; dies ist vergleichbar mit den Baden-Württembergischen Daten, wo im gleichen Zeitraum die Rate bei 0,97 liegt. Bei der Differenzierung fetalen Mortaliät nach Gestationsalter wird klar, dass die Fallzahl aus Baden-Württemberg in den Wochen ab $41+6$ bei den dann noch verbliebenen Schwangeren in diesem Zeitraum höhere Schwankungsbreiten aufweist als das bundesdeutsche Gesamtkollektiv über den größeren Zeitraum. Auch liegt die Rate an gesicherten Übertragungen in Baden-Württemberg mit 0,95\% unter dem Bundesdurchschnitt. Dennoch bestätigen die Bundesdaten die Daten aus Baden-Württemberg, da sie zeigen, dass sich unabhängig von der statistisch zugrunde liegenden Methode eine Erhöhung des Risikos für einen intrauterinen Fruchttod ab $42+0$ nachweisen lässt. Dabei muss im Auge behalten werden, dass das reale Risiko insgesamt nach Überschreiten von vollendeten SSW bis zu $42+6$ mit etwa 2 Fruchttoden je 1000 Geburten insgesamt niedrig, jedoch etwa doppelt so hoch wie im Gestationslater von 37 bis 42 Schwangerschaftswochen liegt. Das gilt für beide Berechnungswege.

Aufgrund der insgesamt niedrigen Fallzahl von fetalen Todesfällen müssen alle beschriebenen Trends vorsichtig interpretiert werden. Eine - wie in der Literatur vorausgesagte - signifikante Senkung der perinatalen Mortalität im Zusammenhang mit einer großzügigeren Einleitungspraxis bereits ab $41+0$ SSW erscheint durch unsere Ergebnisse eher fraglich. Eine detaillierte Auswertung der anderen Risikofaktoren wie bspw. mütterliches Alter, Ernährung, BMI, Rauchen, Parität oder Ethnie aus allen Jahrgängen der Daten der Perinatalerhebung ist für diese Fragestellung erforderlich. Ebenfalls unklar ist es, inwiefern das in Deutschland übliche Prozedere der Schwangerenbetreuung ab 40+0 mit frühzeitigen regelmäßigen Kontrollen der Fruchtwassermenge und der fetalen Herzaktion mittels Ruhe- CTG zu diesen Ergebnissen beiträgt.

\section{Fazit für die Praxis}

$\nabla$

Die vorgestellten Daten zur Geburtseinleitung wurden retrospektiv aus Sekundärdaten entnommen und analysiert. Sie zeigen Zusammenhänge, aber keine Kausalitäten. Dennoch muss festgestellt werden, dass der gewünschte Effekt einer großzügig indizierten Geburtseinleitung an 41+0 Schwangerschaftswochen, nämlich die Reduktion der Totgeburten am Termin, ohne weitere Analysen nicht beweisbar ist. Weitere Forschung, insbesondere eine prospektive Erfassung und Auswertung von Geburtsergebnissen bei Einleitung, ist notwendig. Bis dahin sollte die Empfehlung zur Geburtseinleitung bei Terminüberschreitung ohne weitere Risikofaktoren zurückhaltend und mit der gebotenen Objektivität erfolgen. Die Einbeziehung der betroffenen Frauen in die Entscheidungsfindung muss im Licht der umfassenden Kommunikation der unsicheren wissenschaftlichen Datenlage stattfinden.

\section{Acknowledgements \\ $\nabla$}

I would like to thank EANS and all scholars involved for facilitating the international Summer School for doctoral students on complex interventions. This opportunity was supported by an ESF grant.

\section{Interessenkonflikt \\ $\nabla$}

Es liegen bei keiner der Autorinnen und keinem der Autoren Interessenkonflikte vor.

Institute

Frauenklinik AG Hebammenwissenschaft, Medizinische Hochschule Hannover, Hannover

${ }^{2}$ Frauenklinik, Klinikum Sindelfingen-Böblingen, Böblingen

${ }^{3}$ Department Gesundheit, ZHAW Zürcher Hochschule für Angewandte Wissenschaften, Winterthur, Switzerland

${ }^{4}$ BSC Hebammenkunde, Hochschule für Gesundheit, Bochum

${ }^{5}$ AQUA, Institut für angewandte Qualitätsförderung und Forschung im Gesundheitswesen, Göttingen

${ }^{6}$ Fakultät für Gesundheit Department für Humanmedizin Institut für Integrative Medizin Gerhard Kienle Lehrstuhl für Medizintheorie, Integrative und Anthroposophische Medizin, Universität Witten/Herdecke, Herdecke

\section{Literatur}

1 Gülmezoglu AM, Crowther CA, Middleton P et al. Induction of labour for improving birth outcomes for women at or beyond term. Cochrane database Syst Rev 2012; 6: CD004945 Available from: http://www. ncbi.nlm.nih.gov/pubmed/22696345

2 National Collaborating Centre for Women's and Children's Health. Induction of labour. London: National Institute for Health and Clinical Excellence (NICE), 2008

3 Stock SJ, Ferguson E, Duffy A et al. Outcomes of elective induction of labour compared with expectant management: population based study. BMJ 2012; 344: e2838 Available from http://www.pubmedcentral. nih.gov/articlerender.fcgi? artid $=3349781$ \&tool $=$ pmcentrez\&render type $=$ abstract

4 Wennerholm $U-B$, Hagberg $H$, Brorsson $B$ et al. Induction of labor versus expectant management for post-date pregnancy: is there sufficient evidence for a change in clinical practice? Acta Obstet Gynecol Scand 2009; 88: 6-17 Available from http://www.ncbi.nlm.nih.gov/ pubmed/19140042

5 Grivell RM, Reilly AJ, Oakey $\mathrm{H}$ et al. Maternal and neonatal outcomes following induction of labor: a cohort study. Acta Obstet Gynecol Scand 2012; 91: 198-203 Available from: http://www.ncbi.nlm.nih. gov/pubmed/21995778

6 Kiesewetter B, Lehner R. Maternal outcome monitoring: induction of labor versus spontaneous onset of labor - a retrospective data analysis. Arch Gynecol Obstet 2012; 286: 37-41 Available from: http:// www.ncbi.nlm.nih.gov/pubmed/22298204

7 Khireddine I, Le Ray C, Dupont $C$ et al. Induction of labor and risk of postpartum hemorrhage in low risk parturients. PLoS One 2013; 8: e54858 Available from http://www.plosone.org/article/in fo:doi/10.1371/journal.pone.0054858\#pone-0054858-g001

8 Raviraj P, Shamsa A, Bai J et al. An analysis of the NSW Midwives Data Collection over an 11-year period to determine the risks to the mother and the neonate of induced delivery for non-obstetric indication at term. ISRN Obstet Gynecol 2013; 2013: 178415 Available from http://www.pubmedcentral.nih.gov/articlerender.fcgi?ar tid $=3800658 \&$ tool $=$ pmcentrez\&rendertype $=$ abstract

9 O'Cathain A, Thomas K, Walters SJ et al. Women's perceptions of informed choice in maternity care. Midwifery 2002; 18: 136-144 Available from http://www.ncbi.nlm.nih.gov/pubmed/12139911

10 Elwyn G, Scholl I, Tietbohl C et al. "Many miles to go ...": a systematic review of the implementation of patient decision support interventions into routine clinical practice. BMC Med Inform Decis Mak 2013; 13: S14 Available from http://www.biomedcentral.com/1472$6947 / 13 / \mathrm{S} 2 / \mathrm{S} 14$

11 Hall HG. Complementary and alternative medicine for induction of labour. Women \& Birth (WOMEN BIRTH) 2012 
12 Yudkin PL, Wood L, Redman CWG. Risk of unexplained stillbirth at different gestational ages. Lancet 1987; 329: 1192-1194 Avai lable from http://www.sciencedirect.com/science/article/pii/ S0140673687921544

13 Joseph KS. Theory of obstetrics: an epidemiologic framework for justifying medically indicated early delivery. BMC Pregnancy Childbirth 2007; 7: 4 Available from http://www.pubmedcentral.nih.gov/ articlerender.fcgi? artid $=1851971$ \&tool $=$ pmcentrez\&rendertype $=a b$ stract

14 Smith GC. Life-table analysis of the risk of perinatal death at term and post term in singleton pregnancies. Am J Obstet Gynecol 2001; 184: 489-496 Available from: http://www.sciencedirect.com/science/ article/pii/S0002937801114353
15 Weiss E, Krombholz K, Eichner M. Fetal mortality at and beyond term in singleton pregnancies in Baden-Wuerttemberg/Germany 2004-2009. Arch Gynecol Obstet 2014; 289: 79-84 Available from: http://www. pubmedcentral.nih.gov/articlerender.fcgi? artid $=3889812 \&$ tool $=$ pm centrez\&rendertype $=$ abstract

16 Stock SJ, Ferguson E, Duffy A et al. Outcomes of elective induction of labour compared with expectant management: population based study. BMJ 2012; 344: e2838 Available from http://www.bmj.com/ content/344/bmj.e2838.pdf\%2Bhtml

17 Caughey $A B$, Sundaram V, Kaimal AJ et al. Systematic review: elective induction of labor versus expectant management of pregnancy. Ann Intern Med 2009; 151: 252-263 W53-W63 Available from: http:// www.ncbi.nlm.nih.gov/pubmed/19687492 